\title{
The CTP-binding domain is disengaged from the DNA-binding domain in a co-crystal structure of Bacillus subtilis Noc-DNA complex
}

\author{
Kirill V. Sukhoverkov ${ }^{1 \dagger}$, Adam S. B. Jalal ${ }^{1,2+}$, David M. Lawson ${ }^{3}$, and Tung B. K. Le ${ }^{1}$ \\ ${ }^{1}$ Department of Molecular Microbiology \\ John Innes Centre, Norwich, NR4 7UH, United Kingdom \\ ${ }^{2}$ Section of Structural and Synthetic Biology, Department of Infectious Diseases, Faculty of \\ Medicine, Imperial College London, London, SW7 2AZ, UK \\ ${ }^{3}$ Department of Biochemistry and Metabolism \\ John Innes Centre, Norwich, NR4 7UH, United Kingdom \\ ${ }^{\dagger}$ these authors contributed equally to this work \\ ${ }^{*}$ Correspondence: tung.le@jic.ac.uk
}

\begin{abstract}
In Bacillus subtilis, a ParB-like nucleoid occlusion protein (Noc) binds specifically to Noc-binding sites (NBS) around the chromosome to help coordinate chromosome segregation and cell division. Noc does so by binding to cytidine triphosphate (CTP) to form large membrane-associated nucleoprotein complexes to physically inhibit the assembly of the cell division machinery. The sitespecific binding of Noc to NBS DNA is a prerequisite for CTP-binding and the subsequent formation of a membrane-active DNA-entrapped protein complex. Here, we solve the structure of a truncated $B$. subtilis Noc bound to NBS DNA to reveal the conformation of Noc at this crucial step. Our structure reveals the disengagement between the $\mathrm{N}$-terminal CTP-binding domain and the NBS-binding domain of each DNA-bound Noc subunit, this is driven, in part, by the swapping of helices 4 and 5 at the interface of the two domains. Site-specific crosslinking data suggest that this conformation of Noc-NBS exists in solution. Overall, our results lend support to the recent proposal that parS/NBSbinding catalyzes CTP-binding and DNA-entrapment by preventing the re-engagement of the NTD and DBD from the same ParB/Noc subunit.
\end{abstract}

\section{INTRODUCTION}

Cells must couple chromosome segregation and division to reproduce efficiently. In Firmicutes, such as Bacillus subtilis, the nucleoid occlusion protein Noc contributes to the coordination between chromosome segregation and the initiation of cell division ${ }^{1-}$ 4. Noc helps direct the assembly of the cell division machinery towards the middle of a dividing cell where the concentration of DNA is the least, thus increasing cell division efficiency ${ }^{2-4}$. Critical to this function of Noc is its ability to recruit chromosomal DNA to the cell membrane to form large Noc-DNAmembrane complexes which inhibit the FtsZ formation over the nucleoid and/or to corral the FtsZ ring towards the mid-cell position ${ }^{5,6}$. Noc is a paralog of a chromosome partitioning protein ParB, and is also a CTPase enzyme that binds cytidine triphosphate (CTP) to form a protein clamp that can slide and entrap DNA $^{6-8}$. Apo-Noc first binds to nucleate on 16bp NBS (Noc-binding site) sites scattering along the chromosome $e^{6,9,10}$. The nucleation at
NBS promotes CTP-binding and the subsequent engagement of N-terminal domains from opposing subunits of a Noc homodimer to form a clamp-closed complex that can escape from NBS to slide and spread to the neighboring DNA while still entrapping DNA $^{6}$. The DNA-entrapped Noc-CTP complexes are also active at binding to the cell membrane due to the liberation of a 10-aminoacid membrane-targeting amphipathic helix ${ }^{6}$. As a result, Noc-CTP brings the entrapped chromosomal DNA close to the cell membrane to form large Noc-DNA-membrane complexes that are inhibitory to the assembly of nearby cell division machinery ${ }^{5,6}$.

Previously we solved two X-ray crystallography structures of the CTP-binding domain and DNA-binding domain of a Geobacillus thermoleovorans Noc to better understand the molecular mechanism of this protein family ${ }^{6}$. Nevertheless, it remains unclear how the Noc-NBS binding event mechanistically promotes the $\mathrm{N}$-terminal 
domain engagement to form a closed-clamp Noc. To investigate further, in this study, we solve a structure of a $B$. subtilis Noc-NBS DNA complex to reveal the conformation of $a$ nucleating Noc. Through comparisons to other available structures of Noc, and its paralog ParB, and by in-solution site-specific crosslinking, we provide evidence for the extended conformation of nucleating Noc.

\section{RESULTS}

Co-crystal structure of $B$. subtilis Noc with NBS DNA reveals that the $\mathrm{N}$-terminal CTPbinding domain of each Noc subunit is disengaged from its DNA-binding domain

To gain insight into the nucleating state of Noc, we sought to determine a co-crystal structure of a Noc-NBS complex from $B$. subtilis. After screening several constructs with various lengths of Noc and NBS, we solved a $2.9 \AA$ resolution crystal structure of $B$. subtilis Noc $\triangle C T D$ in complex with 16-bp NBS DNA duplex (see Materials and Methods) (Table 1). The Noc $\triangle C T D$ variant lacks the 41-amino-acid C-terminal domain (CTD) responsible for NoC dimerization (Figure 1A-B) ${ }^{5,6}$. The asymmetric unit contains two copies of Noc $\triangle C T D$ bound to a single 16-bp NBS DNA duplex (Figure 1B).

Each Noc $\triangle$ CTD subunit consists of an Nterminal CTP-binding domain (NTD) (helices a1 to 4 and sheets $\beta 1$ to 4 ) and a DNA-binding domain (DBD) (helices a5 to 11) (Figure 1C). The electron density for the first 27 amino acids that contains the membrane-targeting peptide was poorly resolved, and thus this region was absent from the model (Figure $1 A$ ). Each Noc $\triangle$ CTD subunit is bound to a half NBS site, the NBS DNA adopts a conformation whereby in one strand the 5' base was flipped out, and in the other, the 3' base was flipped out, enabling a sticky-ended interaction (with a one-base overhang) between the duplexes in adjacent asymmetric units (Figure $1 \mathrm{~B}$ and Supplementary Figure 1). We previously solved a structure of only the DBD of Noc with NBS (2.23A, PDB: 6Y93) to elucidate the molecular basis for NBS-binding specificity ${ }^{10}$. Given that the conformation of the DBD and the core NBS site are similar between the previous structure and the structure in this work (root-mean-square deviation RSMD = $0.46 \AA)$, we describe the conformation of the NTD in-depth here instead. By structural alignment of the two Noc $\triangle$ CTD subunits, we noted that the DBD and helices $\alpha 4-5$ are highly similar (RSMD $=0.27 \AA$ ) while the rest of the NTD $(\beta 1-\beta 4)$ is orientated in a different direction (approx. $30^{\circ}$ apart, owing to the flexible loop in between $\alpha 4$ and $\beta 4$ ) (Figure $2 \mathrm{~A})$. The multiple alternative orientations at the NTD is likely a common feature of all nucleating ParB family proteins, including Noc. This was the case for the NTD of Caulobacter crescentus ParB bound to parS DNA ${ }^{11}$, and is also evidential from the superimposition of the $B$. subtilis Noc $\triangle$ CTD-NBS structure onto that of ParB $\Delta$ CTD-parS from Helicobacter pylori and $C$. crescentus (Figure 2B) $)^{11,12}$. Multiple alternative conformations of nucleating $\mathrm{ParB} / \mathrm{Noc}$ family members suggest flexibility at the N-terminal CTP-binding domain.

The most notable feature of the Noc $\Delta$ CTDNBS structure is the disengagement of the NTD and DBD (Figure 1C), which is likely driven by the swinging-out conformation of $\alpha 4$ a5 (Figure 3A-B). Helices $\alpha 4$ and $\alpha 5$ from the same Noc $\triangle C T D$ subunit are not packed together, instead a4 swings outwards by approx. $100^{\circ}$ to pack against $\alpha 5^{\prime}$ from the adjacent Noc $\triangle$ CTD subunit (Figure $3 A$ ). This swinging-out conformation has not been observed in the previous structures of DNAbound $C$. crescentus or $H$. pylori ParB $\triangle$ CTD, of Thermus thermophilus ParB $\Delta$ CTD-apo, or G. thermoleovorans Noc $\Delta$ CTD-apo (Figure 3B and Supplementary Figure 2) $)^{6,11-13}$. In previous structures of apo- or DNA-bound $\mathrm{ParB} / \mathrm{Noc}$, the equivalent helix a4 consistently folds back to pack with a5 from the same protein subunit (the folding-back conformation) (Figure 3B and Supplementary Figure 2). The swinging-out conformation of helices $\alpha 4-5$ is often associated with the nucleotide-bound state of ParB/Noc instead (Figure $3 \mathrm{~B}$ and Supplementary Figure $2)^{6,7,11,14}$. It has been suggested that CTPbinding most likely facilitates the swinging-out conformation of $\mathrm{ParB} / \mathrm{Noc}$ since nucleotides have been observed to make numerous contacts to both the equivalent $\alpha 4$ and the $\alpha 4$ a5 connecting loop in various ParB proteins ${ }^{7,8,11,14}$. The observation of a swingingout conformation in DNA-bound Noc is therefore surprising, given that CTP was not included in the crystallization drop and that CTP-binding is incompatible with high-affinity binding at the nucleation site $N B S^{6}$. We reason that the swinging-out conformation might be 
thermodynamically possible in the DNA-bound nucleating ParB/Noc, and that CTP-binding, instead of facilitating, further stabilizes the swinging-out conformation.

\section{Site-specific \\ cysteine-cysteine crosslinking suggests the swinging-out conformation of Noc-NBS in solution}

To test if the swinging-out conformation of a4a5 is possible in NBS-bound Noc in solution, we employed site-specific chemical crosslinking with the cysteine-specific bismaleimide compound $\mathrm{BMOE}^{15}$. Based on the structures of apo-Noc $\Delta$ CTD $^{6}$ and Noc $\Delta$ CTDNBS, we engineered a dual cysteine substitution at E112 and $\mathrm{H} 143$ on an otherwise cysteine-free $B$. subtilis Noc to create a Noc (E112C H143C) variant (Figure 3A). In the folding-back conformation where helices a4 and $\alpha 5$ from the same Noc subunit pack together, crosslinking of E112C to H143C would generate an intramolecular crosslinked species (Noc IntraXL), while a swinging-out conformation would give rise to intermolecularly crosslinked species (a singlycrosslinked Noc InterXL and a doublycrosslinked Noc Inter2XL) which are twice the theoretical molecular weight of a Noc monomer (Figure 4A). Crosslinking of apoNoc (E112C H143C) only resulted in a prominent band that migrated faster in a denaturing acrylamide gel than noncrosslinked protein (Figure 4B, lane 1 vs. 2), this is most likely a Noc IntraXL species. Little of Noc InterXL or Inter2XL species was observed ( $4.4 \%$ crosslinked fraction) suggesting that the swinging-out conformation is unfavored in apo-Noc (Figure 4B, lane 1 vs. lane 2). The addition of only CTP did not promote the swinging-out conformation noticeably (Figure $4 \mathrm{~B}$, lane $2,-4.4 \%$ vs. lane $4,-8.7 \%$ crosslinked fraction). The singly (InterXL) and the doubly (Inter2XL) crosslinked species appeared more prominently when NBS only (Figure 4B, lane $2,-4.4 \%$ vs. lane $3,-19.3 \%$ crosslinked fraction) or NBS + CTP were preincubated with Noc (Figure 4B, lane 2, $-4.4 \%$ vs. lane 5 , $-31.5 \%$ crosslinked fraction). The InterXL/2XL fraction further increased when NBS was used in a molar excess to Noc (E112C H143C) (Supplementary Figure 3). We were able to assign different bands to either being InterXL or Inter2XL by performing crosslinking reactions of Noc (E112C H143C) + NBS +
CTP with an increasing concentration of the BMOE crosslinker (Figure 4C). The assumption is that a singly-crosslinked InterXL preferably forms at a lower concentration of a crosslinker. Overall, our result here suggests that the swinging-out conformation of a4-5 is possible in solution and is promoted when Noc is bound to the NBS DNA.

\section{DISCUSSION}

In $B$. subtilis, noc resulted from parB via a gene duplication and neo-functionalization event ${ }^{10,16}$, and both Noc and ParB are CTPdependent molecular switches $7,8,17-20$. CTPbinding switches nucleating ParB/Noc (bound at a high-affinity parS/NBS site) from an openclamp conformation (Figure 5A-B) to a closedclamp conformation that can escape from parS/NBS to slide to neighboring DNA while still entrapping DNA (Figure 5C) 6,7,14,17,18. The closed-clamp conformation is possible due to the new dimerization interface between the two adjacent $\mathrm{N}$-terminal CTP-binding domains of ParB/Noc (the so-called NTD-NTD engagement, Figure $5 C)^{6,7,14,17}$. Here, our Noc $\triangle$ CTD-NBS structure represents an openclamp conformation because there is no protein-protein contact between the majority of two adjacent NTDs of Noc, except for the swapping helices $\alpha 4$ and $\alpha 4$ ' (Figure $1 \mathrm{~B}$ and Figure 5B).

It has been observed that, without parS/NBS, CTP is unable to efficiently promote the NTDNTD engagement to close the ParB/Noc clamp $p^{6,7,14,17}$. To rationalize this phenomenon, Antar et al (2021) noted that two ParB subunits would not be able to occupy a pars site if they were to adopt a conformation similar to apoParB (in which the NTD and the DBD of the same ParB subunit fold back on each other) because of a severe clash between opposing ParB subunits ${ }^{15}$. Antar et al (2021) proposed that, to avoid this potential clash, the NTD and the DBD from each parS-bound ParB must be untethered/disengaged from each other ${ }^{15}$. The DBD-NTD disengagement later favors the two opposing NTDs to dimerize in the presence of CTP to form a clamp-closed complex ${ }^{15}$. In sum, parS serves a catalyst in a reaction that favors the formation of the product (the closed clamp) by inhibiting the reversion to the substrate (the open clamp apo-ParB). Our structure of DNA-bound Noc here lends support to this hypothesis because the 
conformation of the DNA-bound Noc subunit is drastically different from that of apo-Noc, especially with the swinging-out helices a4-a5 disengaging the NTD and DBD from each other (Figure $5 \mathrm{Bi}$ ). It is possible that NBSbound Noc might exist as an ensemble of states with helices $\alpha 4-\alpha 5$ in either a foldingback (Figure 5Bii) or a swinging-out conformation (Figure 5Bi), and that the Noc $\triangle$ CTD-NBS structure here represents a snapshot of this dynamic process. The swinging-out conformation of a4-a5 might be rare in solution, given that the crosslinking reaction of Noc (E112C H143C) + NBS produced IntraXL as the major species. Nevertheless, the proportion of Inter $X L$ and Inter2XL increased substantially when NBS (Figure 4B, lane 3 ) is included in comparison to apo-Noc only (lane 2) or Noc + CTP only conditions (lane 4). Moreover, the proportion of InterXL and Inter2XL also increased when NBS was added in excess (Supplementary Figure 3). The proximity of adjacent Noc subunits and the restriction in movement by a DNA-fixated DBD may increase the likelihood of swapping helices $\alpha 4-\alpha 5$ in Noc. This might in part contribute to further promoting the NTD-NTD engagement upon CTP-binding (Figure 5C), and might additionally explain how NBS serves as a catalysis for NTD-NTD engagement and thus clamp closure for a ParB-like protein Noc. However, it is also worth noting that ParB, in the presence of parS, does not undergo a4-a5 swapping as readily as Noc-NBS (S. Gruber, personal communication $)^{15}$. It is still unclear why this is the case and how it is related to the biological functions of ParB vs. Noc, but it explains why helices $\alpha 4-\alpha 5$ in all previous $X$-ray crystallography structures of ParB-parS complex are all in the folding-back conformation ${ }^{11,12}$.

\section{ACKNOWLEDGEMENTS}

This study was funded by the Royal Society University Research Fellowship Renewal (URFIRI201020 to T.B.K.L) and BBSRC (BBS/E/J/000PR9791 to the John Innes Centre). K.V.S is supported by Wellcome grant (221776/Z/20/Z). A.S.B.J's PhD studentship was funded by the Royal Society (RG150448). We thank Diamond Light Source for access to beamline 104 under proposal MX18565. We thank Clare Stevenson for assistance with X-ray crystallography. We thank S. Gruber, H. Antar, and T. McLean for discussions and comments on the manuscript.

\section{MATERIALS AND METHODS Plasmid and strain construction}

Construction of pET21b:: Bacillus subtilis NocACTD-his 6 and pET21b::noc (E112C H143C)-his 6

The coding sequence of a 41-amino-acid Cterminally truncated $B$. subtilis Noc was amplified by PCR using a forward primer (aactttaagaaggagatatacatatgaagcattcattctctcg tttcttc) and a reverse primer (gtggtgctcgagtgcggccgcaagcttatctctgctgaatgc tttgcgtctc), and pET21b::B. subtilis Noc-his ${ }_{6}{ }^{6}$ as template. The resulting PCR product was gel-purified and assembled into an NdelHindIII-cut pET21b using a 2x Gibson master mix (NEB). Gibson assembly was possible owing to a 23-bp sequence shared between the Ndel-and-HindIII cut pET21b backbone and the PCR amplified fragment. The 23-bp homologous region was introduced during the synthesis of the above primers.

A double-stranded DNA (dsDNA) fragment containing a $B$. subtilis noc (E112C H143C) gene was chemically synthesized (gBlocks, IDT). The gBlocks fragment was assembled into an Ndel-Hindlll-cut pET21b using a $2 x$ Gibson master mix to result in $p E T 21 b:: n o c$ (E112C H143C)-his6. All plasmids were verified by Sanger sequencing (Eurofins, Germany).

\section{Protein overexpression and purification}

$B$. subtilis Noc $\Delta C T D-\mathrm{His}_{6}$ were purified through a 3-column (HisTrap, Heparin, Superdex-75 gel filtration) procedure as described previously ${ }^{6}$. Purified Noc $\Delta$ CTD-His 6 was stored at $-80^{\circ} \mathrm{C}$ in storage buffer $(10 \mathrm{mM}$ Tris- $\mathrm{HCl} \mathrm{pH} 8.0$ and $250 \mathrm{mM} \mathrm{NaCl}$ ) before crystallization.

Noc (E112C H143C)-His 6 was purified through a 2-column (His-Select Cobalt Affinity Gel, Superdex-200 gel filtration) procedure using the following buffers: buffer A-HisTrap (100 $\mathrm{mM}$ Tris- $\mathrm{HCl} \mathrm{pH} 7.4,300 \mathrm{mM} \mathrm{NaCl}, 10 \mathrm{mM}$ imidazole, $5 \%$ (v/v) glycerol), buffer B-HisTrap (100 mM Tris- $\mathrm{HCl}$ pH 7.4, $300 \mathrm{mM} \mathrm{NaCl}, 500$ $\mathrm{mM}$ imidazole, 5\% (v/v) glycerol), and gel filtration buffer $(100 \mathrm{mM}$ Tris- $\mathrm{HCl} \mathrm{pH} 7.4$ and $300 \mathrm{mM} \mathrm{NaCl}$ ). Purified protein was concentrated using an Amicon Ultra-4 10 kDa 
cut-off spin column, and stored at $-80^{\circ} \mathrm{C}$ in storage buffer $(100 \mathrm{mM}$ Tris- $\mathrm{HCl} \mathrm{pH} 7.4,300$ $\mathrm{mM} \mathrm{NaCl}, 10(\mathrm{v} / \mathrm{v})$ glycerol, and $0.1 \mathrm{mM}$ TCEP).

\section{In vitro crosslinking using a sulfhydryl-to- sulfhydryl crosslinker bismaleimidoethane (BMOE)}

Noc (E112C H143C)-His $6 \quad(4 \mu M \quad$ final concentration) was incubated on ice either alone or with $1 \mathrm{mM}$ CTP, or $1 \mu \mathrm{M}$ 22-bp NBS DNA duplex (or with a twofold increasing concentration of NBS from 0 to $5 \mu \mathrm{M}$ ), or both in a crosslinking buffer $(100 \mathrm{mM}$ Tris- $\mathrm{HCl} \mathrm{pH}$ 7.4, $130 \mathrm{mM} \mathrm{NaCl}, 5 \mathrm{mM} \mathrm{MgCl}$ ) for $10 \mathrm{~min}$. Then, $20 \mathrm{mM}$ DMSO solution of the crosslinking reagent (BMOE, ThermoFisher) was added to the reaction to the final concentration of $2 \mathrm{mM}$. The mixture was incubated at room temperature for $5 \mathrm{~min}$ before the crosslinking reaction was quenched by SDS-PAGE loading dye $+\beta$ mercaptoethanol. Samples were heated to $90^{\circ} \mathrm{C}$ for $10 \mathrm{~min}$ before being loaded on $4-12 \%$ Bis-Tris polyacrylamide gels (ThermoFisher). Each experiment was triplicated. Polyacrylamide gels were stained in an InstantBlue Coomassie solution (Abcam) and band intensity was quantified using Image Studio-Lite (LICOR Biosciences). Raw gel images were deposited to the Mendeley repository: doi: 10.17632/6sp26rm6zy.1

\section{Reconstitution of NBS DNA for X-ray crystallography}

A 16-bp NBS DNA fragment (5'TATTTCCCGGGAAATA-3') (3.6 mM in buffer containing $10 \mathrm{mM}$ Tris- $\mathrm{HCl} \mathrm{pH} 8.0$ and 250 $\mathrm{mM} \mathrm{NaCl}$ ) was heated to $98^{\circ} \mathrm{C}$ for 5 min before being left to cool at room temperature overnight to form double-stranded NBS DNA (final concentration: $1.8 \mathrm{mM}$ ).

\section{Protein crystallization, structure determination, and refinement}

B. subtilis Noc $\Delta$ CTD-His $6(\sim 10 \mathrm{mg} / \mathrm{mL})$ was mixed with the 16-bp NBS DNA at a molar ratio of $1: 1.2$ (protein:DNA) in the gel filtration elution buffer (10 mM Tris- $\mathrm{HCl}$ pH 8.0, $250 \mathrm{mM}$ $\mathrm{NaCl})$. Crystallization screens were set up in sitting-drop vapor diffusion format in MRC2 96-well crystallization plates with drops comprised of $0.3 \mu \mathrm{L}$ precipitant solution and $0.3 \mu \mathrm{L}$ of protein and incubated at $293 \mathrm{~K}$. After optimization of initial hits, the best crystals of the complex grew in a solution containing $17 \%$ (w/v) PEG3350, $0.25 \mathrm{M}$ magnesium acetate and $10 \% \quad(\mathrm{v} / \mathrm{v})$ sucrose. These were cryoprotected in the crystallization solution supplemented with $20 \%(\mathrm{v} / \mathrm{v})$ glycerol and mounted in Litholoops (Molecular Dimensions) before flash-cooling by plunging into liquid nitrogen. X-ray data were recorded on beamline 104 at the Diamond Light Source (Oxfordshire, UK) using an Eiger2 XE 16M hybrid photon counting detector (Dectris), with crystals maintained at $100 \mathrm{~K}$ by a Cryojet cryocooler (Oxford Instruments). Diffraction data were integrated and scaled using DIALS $^{21}$ via the XIA2 expert system ${ }^{22}$ then merged using AIMLESS ${ }^{23}$ to a resolution of $2.9 \AA$ in space group $P 22_{1} 2_{1} 2_{1}$ with cell parameters of $a=70.5, b=99.3, c=99.4 \AA$. Data collection statistics are summarized in Table 1. Analysis of the likely composition of the asymmetric unit (ASU) suggested that it contained two copies of the $29.5 \mathrm{kDa}$ Noc $\triangle$ CTD monomer plus the 16-bp NBS duplex, giving an estimated solvent content of $51 \%$.

The majority of the downstream analysis was performed through the CCP4i2 graphical user interface $^{24}$. For molecular replacement, a template was constructed from the structure of the $B$. subtilis NOC DNA-binding domain (DBD) complexed to an NBS duplex (PDB accession code $6 Y 93)^{10}$. Initially, PHASER ${ }^{25}$ was run using the protein and DNA components of this entry comprising two copies of the DBD and one DNA duplex, although the latter was truncated from a $22 \mathrm{mer}$ to a 16mer. This yielded a good solution and, in common with the template structure, the DNA formed a pseudo-continuous filament spanning the crystal due to base-pair stacking between DNA fragments in adjacent ASUs. However, there was only sufficient space to accommodate $15 \mathrm{bp}$ per ASU within this filament. For the time being, the DNA model was truncated to the central $14 \mathrm{bp}$ NBS site in $\mathrm{COOT}^{26}$ before real space refining using "chain refine". The model was subsequently refined with REFMAC5 ${ }^{27}$, using jelly body refinement giving $R_{\text {work }}$ and $R_{\text {free }}$ values of 0.363 and 0.404 , respectively, to $2.9 \AA$ resolution. Inspection of the electron density at this stage revealed evidence for the missing $\mathrm{N}$-terminal domains (NTDs). A template for these was generated using SCULPTOR ${ }^{28}$ 
from the Geobacillus thermoleovorans NOC structure (PDB accession code $7 \mathrm{NFU})^{6}$, where the corresponding domain shares $67 \%$ sequence identity with $B$. subtilis. After quickly tidying the output of the REFMAC5 job in COOT, this was put back into PHASER as a search model together with two copies of the NTD template. However, PHASER was only able to place one of the latter sensibly. After further jelly body refinement of this partial model (giving $R_{\text {work }}$ and $R_{\text {free }}$ values of 0.313 and 0.351 , respectively, to $2.9 \AA$ resolution) the electron density was inspected again in COOT, at which point it was possible to manually dock the missing domain into fragmented density. Following restrained refinement in REFMAC5, the density for the DNA was much clearer, enabling the missing DNA bases to be fitted. In one strand the 5' base was flipped out, and in the other, the 3' base was flipped out, enabling a sticky-ended interaction (with a one-base overhang) between the duplexes in adjacent ASUs. After further iterations of model building in COOT and restrained refinement in REFMAC5, the final model was produced with $R_{\text {work }}$ and $R_{\text {free }}$ values of 0.230 and 0.277 , respectively, to 2.9 $\AA$ resolution. Refinement and validation statistics are summarized in Table 1. 


\section{Table 1. X-ray data collection, processing, and refinement statistics}

\begin{tabular}{|c|c|}
\hline \multicolumn{2}{|l|}{ Data collection } \\
\hline Diamond Light Source beamline & 104 \\
\hline Wavelength $(\AA)$ & 0.979 \\
\hline Detector & Eiger2 XE 16M \\
\hline Resolution range $(\AA)$ & $70.27-2.90(3.08-2.90)$ \\
\hline Space Group & $P 212121$ \\
\hline Cell parameters $(\AA)$ & $a=70.5, b=99.3, c=99.4$ \\
\hline Total no. of measured intensities & $164414(27117)$ \\
\hline Unique reflections & $16090(2546)$ \\
\hline Multiplicity & $10.2(10.7)$ \\
\hline Mean $/ / \sigma(I)$ & $11.7(1.2)$ \\
\hline Completeness (\%) & $100.0(100.0)$ \\
\hline$R_{\text {merge }}{ }^{\mathrm{a}}$ & $0.097(1.976)$ \\
\hline$R_{\text {meas }}{ }^{\mathrm{b}}$ & $0.102(2.076)$ \\
\hline $\mathrm{CC}_{1 / 2}^{\mathrm{c}}$ & $0.999(0.691)$ \\
\hline Wilson $B$ value $\left(\AA^{2}\right)$ & 85.6 \\
\hline \multicolumn{2}{|l|}{ Refinement } \\
\hline Resolution range $(\AA)$ & $70.27-2.90(2.98-2.90)$ \\
\hline Reflections: working/free ${ }^{d}$ & $15216 / 1101$ \\
\hline$R_{\text {work }}^{\mathrm{e}}$ & $0.230(0.407)$ \\
\hline$R_{\text {free }} \mathrm{e}$ & $0.278(0.419)$ \\
\hline $\begin{array}{l}\text { Ramachandran plot: } \\
\text { favored/allowed/disallowed }{ }^{f}(\%)\end{array}$ & $96.5 / 3.5 / 0$ \\
\hline R.m.s. bond distance deviation $(\AA)$ & 0.003 \\
\hline R.m.s. bond angle deviation $\left({ }^{\circ}\right)$ & 1.18 \\
\hline Mean $B$ factors: protein/DNA/overall $\left(\AA^{2}\right)$ & $123 / 86 / 117$ \\
\hline PDB accession code & $70 L 9$ \\
\hline
\end{tabular}

Values in parentheses are for the outer resolution shell.

${ }^{\text {a }} R_{\text {merge }}=\sum h k l \sum_{i}\left|l_{i}(h k l)-\langle l(h k l)\rangle\right| / \sum_{h k l} \sum_{i} l_{i}(h k l)$.

${ }^{\mathrm{b}} R_{\text {meas }}=\sum_{h k l}[N /(N-1)]^{1 / 2} \times \sum_{i}\left|l_{i}(h k l)-\langle l(h k l)\rangle\right| / \sum_{k l} \sum_{i} l_{i}(h k l)$, where $l_{i}(h k l)$ is the ith observation of reflection $h k l,\langle l(h k l)\rangle$ is the weighted average intensity for all observations $i$ of reflection $h k l$ and $N$ is the number of observations of reflection $h k l$. ${ }^{c} \mathrm{CC}_{1 / 2}$ is the correlation coefficient between symmetry equivalent intensities from random halves of the dataset.

d The dataset was split into "working" and "free" sets consisting of 95 and $5 \%$ of the data respectively. The free set was not used for refinement.

e The R-factors $R_{\text {work }}$ and $R_{\text {free }}$ are calculated as follows: $R=\sum\left(\left|F_{\text {obs }}-F_{\text {calc }}\right|\right) / \Sigma\left|F_{\text {obs }}\right|$, where $F_{\text {obs }}$ and $F_{\text {calc }}$ are the observed and calculated structure factor amplitudes, respectively.

${ }^{f}$ As calculated using MolProbity ${ }^{29}$ 


\section{REFERENCES}

1. Pang, T., Wang, X., Lim, H. C., Bernhardt, T. G. \& Rudner, D. Z. The nucleoid occlusion factor Noc controls DNA replication initiation in Staphylococcus aureus. PLOS Genetics 13, e1006908 (2017).

2. Wu, L. J. \& Errington, J. Coordination of cell division and chromosome segregation by a nucleoid occlusion protein in Bacillus subtilis. Cell 117, 915-925 (2004).

3. Rodrigues, C. D. A. \& Harry, E. J. The Min System and Nucleoid Occlusion Are Not Required for Identifying the Division Site in Bacillus subtilis but Ensure Its Efficient Utilization. PLOS Genetics 8, e1002561 (2012).

4. Yu, Y., Zhou, J., Gueiros-Filho, F. J., Kearns, D. B. \& Jacobson, S. C. Noc Corrals Migration of FtsZ Protofilaments during Cytokinesis in Bacillus subtilis. mBio 12, (2021).

5. Adams, D. W., Wu, L. J. \& Errington, J. Nucleoid occlusion protein Noc recruits DNA to the bacterial cell membrane. EMBO J. 34, 491-501 (2015).

6. Jalal, A. S. et al. CTP regulates membrane-binding activity of the nucleoid occlusion protein Noc. Mol Cell S1097-2765(21)00505-0 (2021) doi:10.1016/j.molcel.2021.06.025.

7. Soh, Y.-M. et al. Self-organization of parS centromeres by the ParB CTP hydrolase. Science 366, 1129-1133 (2019).

8. Osorio-Valeriano, M. et al. ParB-type DNA Segregation Proteins Are CTP-Dependent Molecular Switches. Cell 179, 1512-1524.e15 (2019).

9. Wu, L. J. et al. Noc protein binds to specific DNA sequences to coordinate cell division with chromosome segregation. EMBO J. 28, 1940-1952 (2009).

10. Jalal, A. S. et al. Diversification of DNA-Binding Specificity by Permissive and SpecificitySwitching Mutations in the ParB/Noc Protein Family. Cell Rep 32, 107928 (2020).

11. Jalal, A. S. et al. A CTP-dependent gating mechanism enables ParB spreading on DNA. Elife 10, e69676 (2021).

12. Chen, B.-W., Lin, M.-H., Chu, C.-H., Hsu, C.-E. \& Sun, Y.-J. Insights into ParB spreading from the complex structure of Spo0J and parS. Proc. Natl. Acad. Sci. U.S.A. 112, 6613-6618 (2015).

13. Leonard, T. A., Butler, P. J. G. \& Löwe, J. Structural analysis of the chromosome segregation protein Spo0J from Thermus thermophilus. Mol. Microbiol. 53, 419-432 (2004).

14. Osorio-Valeriano, M. et al. The CTPase activity of ParB determines the size and dynamics of prokaryotic DNA partition complexes. Mol Cell 81, 3992-4007.e10 (2021).

15. Antar, H. et al. Relief of ParB autoinhibition by parS DNA catalysis and recycling of ParB by CTP hydrolysis promote bacterial centromere assembly. Science Advances (2021) doi:10.1126/sciadv.abj2854.

16. Sievers, J., Raether, B., Perego, M. \& Errington, J. Characterization of the parB-Like yyaA Gene of Bacillus subtilis. J. Bacteriol. 184, 1102-1111 (2002).

17. Jalal, A. S., Tran, N. T. \& Le, T. B. ParB spreading on DNA requires cytidine triphosphate in vitro. eLife 9, e53515 (2020).

18. Balaguer, F. de A. et al. CTP promotes efficient ParB-dependent DNA condensation by facilitating one-dimensional diffusion from parS. eLife 10, e67554 (2021).

19. Walter, J.-C. et al. Physical Modeling of a Sliding Clamp Mechanism for the Spreading of ParB at Short Genomic Distance from Bacterial Centromere Sites. iScience 23, 101861 (2020).

20. Taylor, J. A., Seol, Y., Budhathoki, J., Neuman, K. C. \& Mizuuchi, K. CTP and parS coordinate ParB partition complex dynamics and ParA-ATPase activation for ParABS-mediated DNA partitioning. eLife 10, e65651 (2021).

21. Winter, G. et al. DIALS: implementation and evaluation of a new integration package. Acta Crystallogr D Struct Biol 74, 85-97 (2018).

22. Winter, G. xia2: an expert system for macromolecular crystallography data reduction. $J$ Appl Cryst 43, 186-190 (2010).

23. Evans, P. R. \& Murshudov, G. N. How good are my data and what is the resolution? Acta Cryst D 69, 1204-1214 (2013).

24. Potterton, L. et al. CCP4i2: the new graphical user interface to the CCP4 program suite. Acta Crystallogr D Struct Biol 74, 68-84 (2018).

25. McCoy, A. J. et al. Phaser crystallographic software. J Appl Crystallogr 40, 658-674 (2007). 
bioRxiv preprint doi: https://doi.org/10.1101/2022.02.28.481274; this version posted March 1, 2022. The copyright holder for this preprint (which was not certified by peer review) is the author/funder. All rights reserved. No reuse allowed without permission.

26. Emsley, P. \& Cowtan, K. Coot: model-building tools for molecular graphics. Acta Crystallogr. D Biol. Crystallogr. 60, 2126-2132 (2004).

27. Murshudov, G. N., Vagin, A. A. \& Dodson, E. J. Refinement of Macromolecular Structures by the Maximum-Likelihood Method. Acta Cryst D 53, 240-255 (1997).

28. Bunkóczi, G. \& Read, R. J. Improvement of molecular-replacement models with Sculptor. Acta Crystallogr D Biol Crystallogr 67, 303-312 (2011).

29. Williams, C. J. et al. MolProbity: More and better reference data for improved all-atom structure validation. Protein Sci 27, 293-315 (2018). 

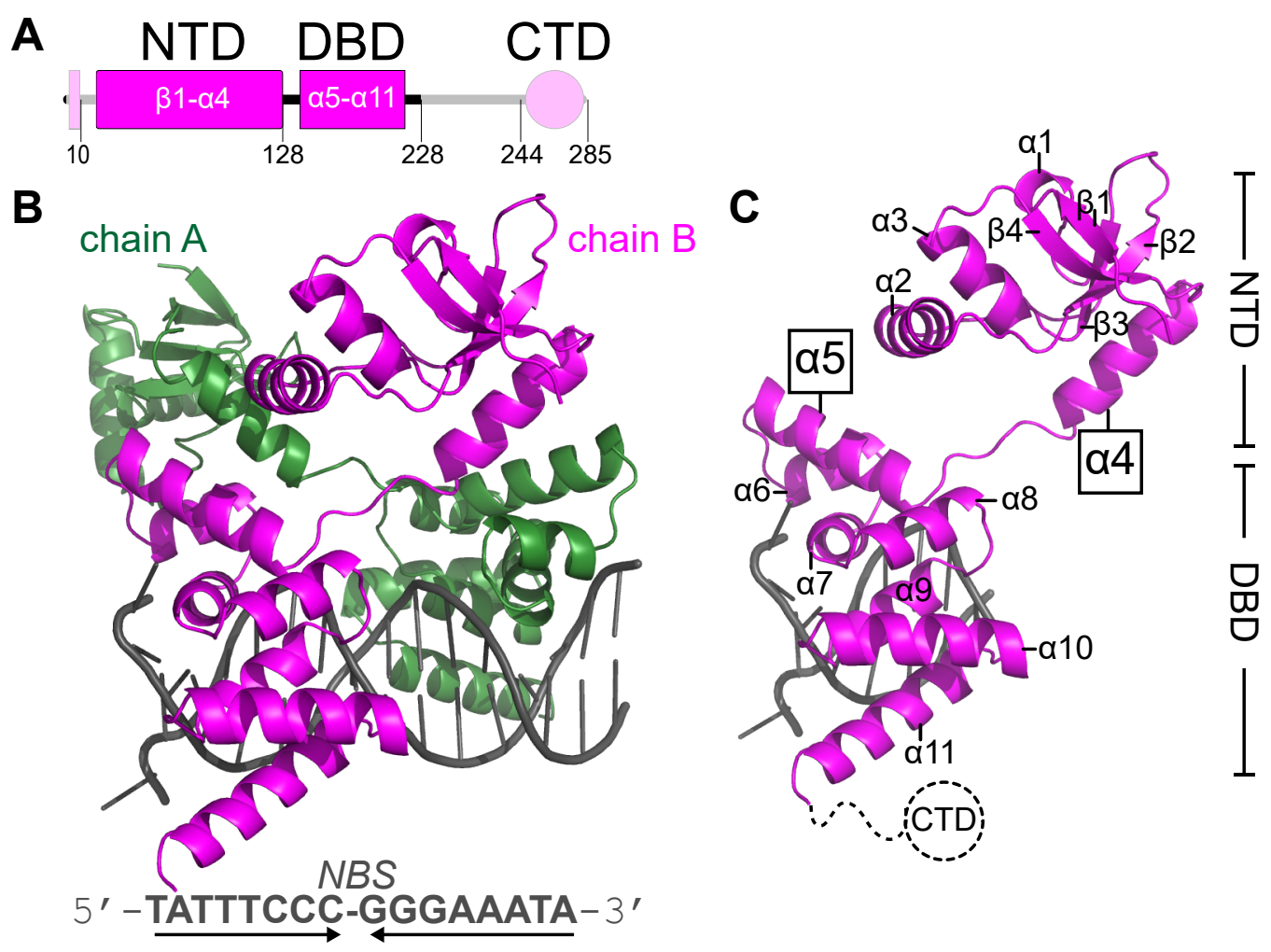

Figure 1. Co-crystal structure of $B$. subtilis Noc with NBS DNA reveals that the $\mathrm{N}$-terminal CTP-binding domain of each Noc subunit is disengaged from its DNA-binding domain. (A) The domain architecture of $B$. subtilis Noc: the 10-amino-acid N-terminal membrane-targeting peptide, the N-terminal CTP-binding domain (NTD), the central DNA-binding domain (DBD), and the C-terminal domain (CTD). Segments of Noc that are not observed in the structure of Noc $\triangle$ CTD-NBS DNA are shown in pale magenta. (B) Co-crystal structure of two Noc $\Delta$ CTD subunits (chain A: dark green, chain B: magenta) bound to a 16-bp NBS DNA duplex (grey). The nucleotide sequence of a 16-bp NBS is shown below the crystal structure, converging arrows indicate that NBS is palindromic. (C) The structure of chain B of Noc $\triangle$ CTD (magenta) bound to an NBS half-site with key features such as the swinging-out helices a4- $\alpha 5$ highlighted. 

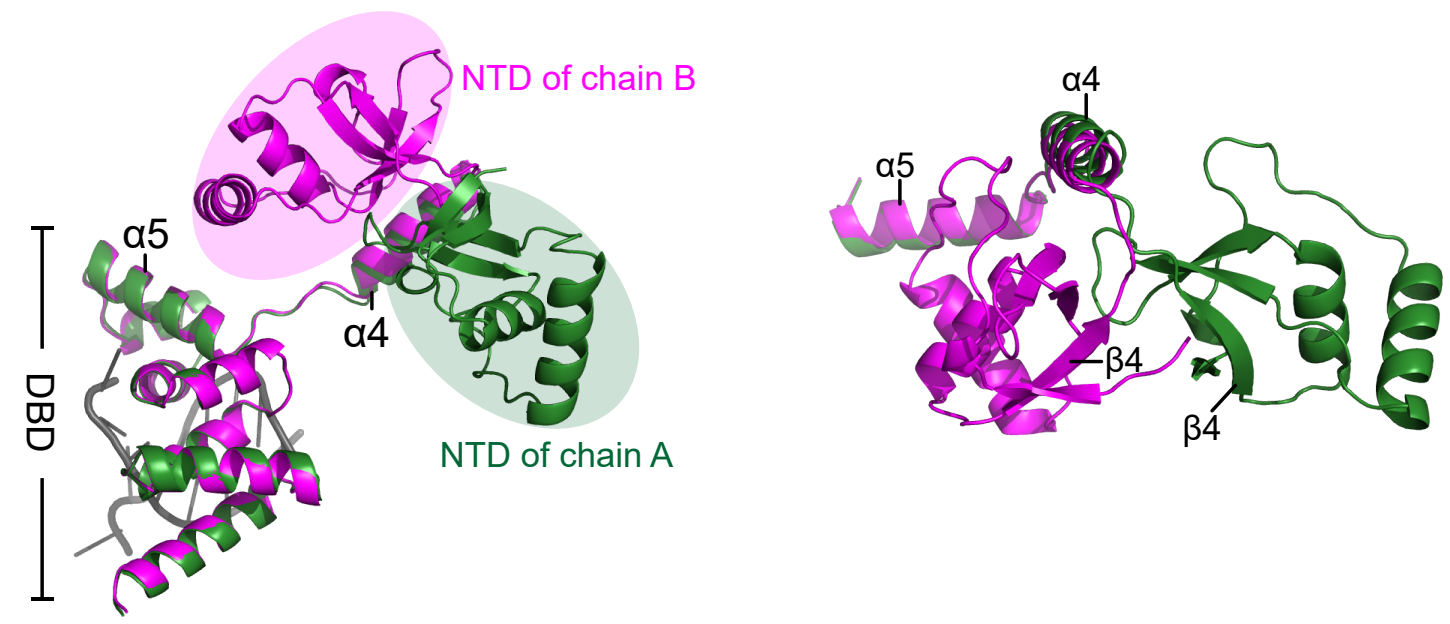

B

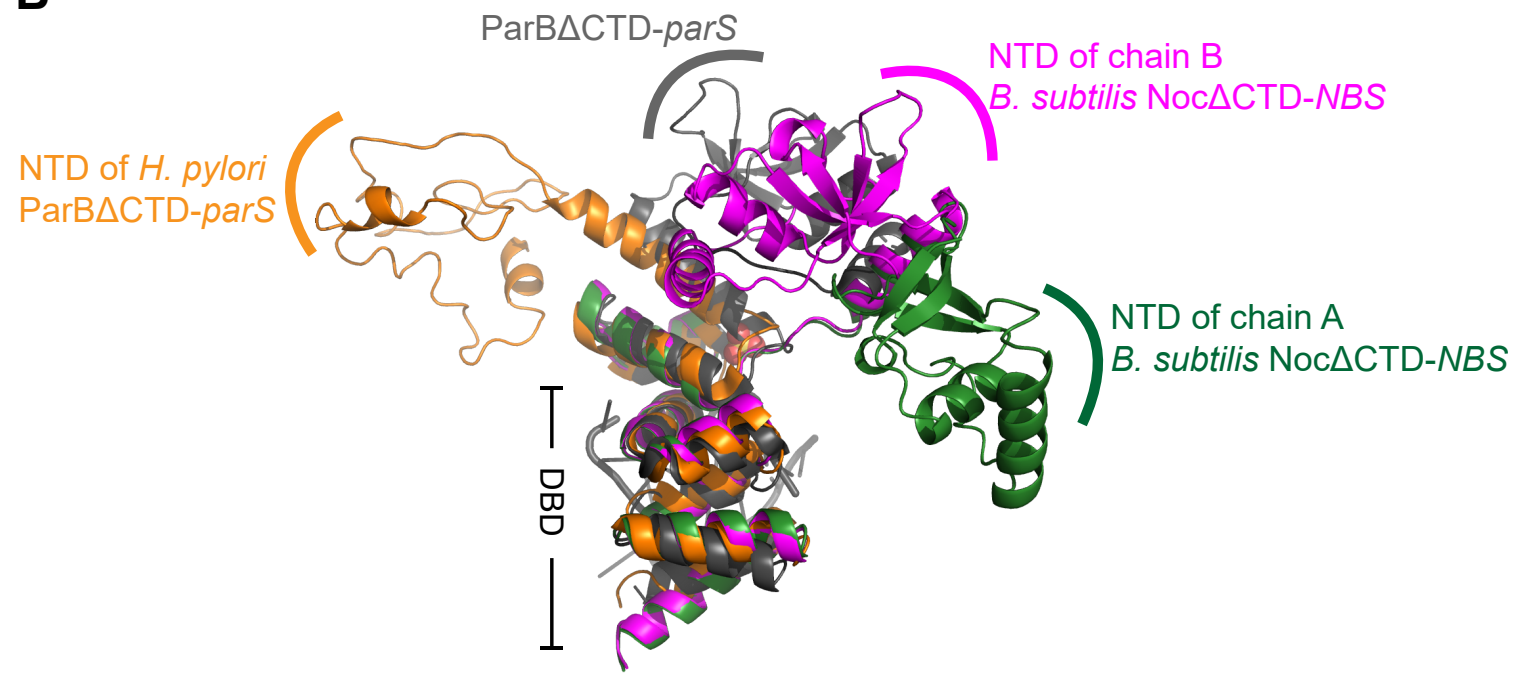

Figure 2. Co-crystal structure of $B$. subtilis Noc $\triangle$ CTD-NBS shows alternative orientations at the N-terminal domain (NTD) of Noc. (A) (left panel) Superimposition of chain A and chain B of Noc $\triangle$ CTD shows the different orientations of the NTD. (right panel) the top-down view of the superimposition of Noc $\triangle$ CTD subunits shows the majority of the NTD orientates $\sim 30^{\circ}$ apart; part of the DBD (from a6 to a11) was omitted for clarity. Half of the 16-bp palindromic NBS site (grey) is shown. (C) Structural superimposition of $B$. subtilis Noc $\triangle$ CTD-NBS upon other available DNA-bound ParB structures highlights the variation in the orientation of the NTD. 


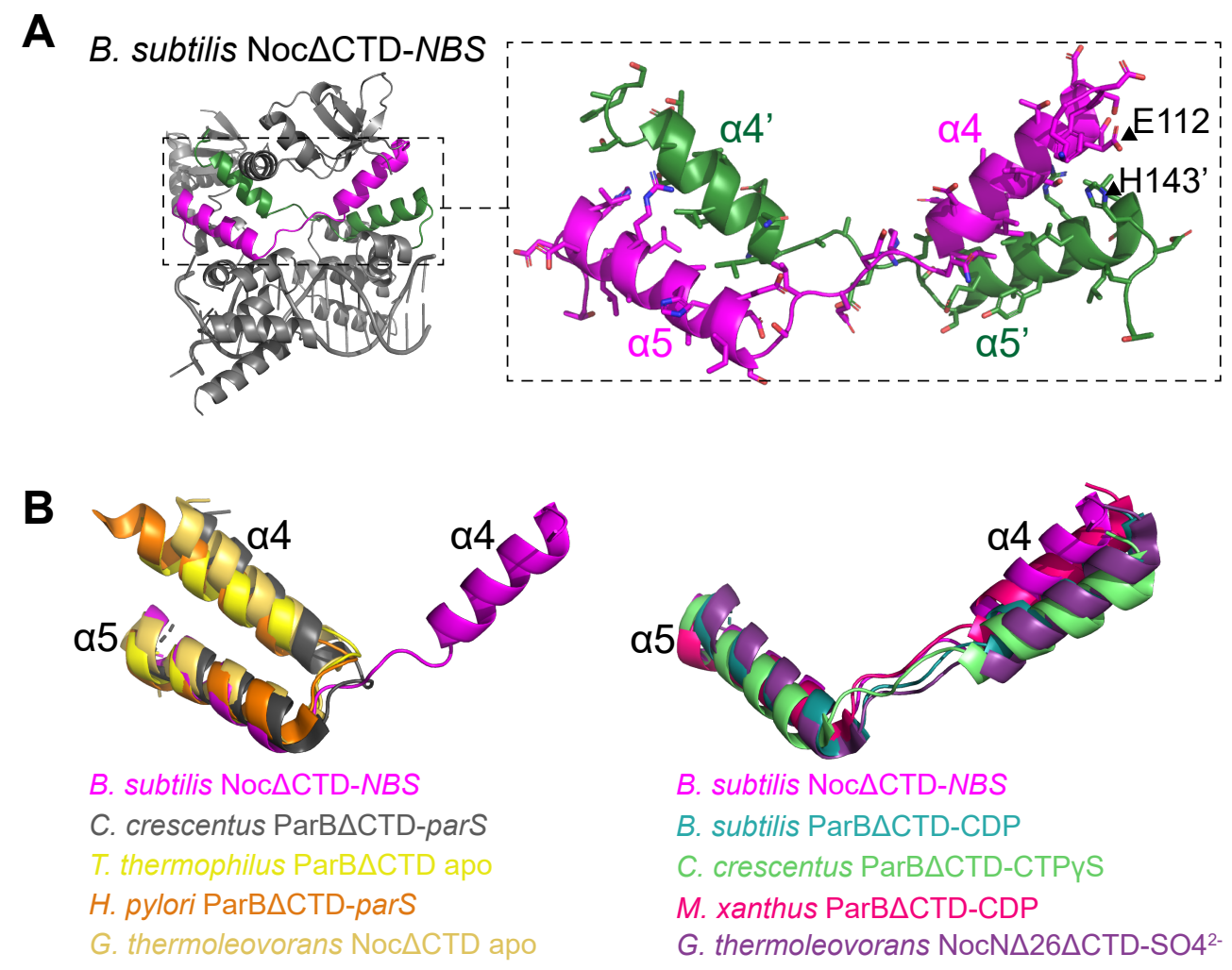

Figure 3. Helices $\alpha 4-\alpha 5$ from the $B$. subtilis Noc $\triangle$ CTD-NBS complex adopt a swinging-out conformation. (A) Co-crystal structure of $B$. subtilis Noc $\triangle C T D$ with NBS (left panel) with the pairs of swapping helices ( $\alpha 4-\alpha 5$, and $\alpha 4$ '- $\alpha 5$ ' for the opposite subunit) highlighted in magenta and dark green, respectively (right panel). Amino acid side chains of $\alpha 4-\alpha 5$ and $\alpha 4$ '- $\alpha 5$ ' are shown in stick representation to illustrate the packing between helices from opposite Noc subunit. The positions of residues $\mathrm{E} 112$ (on a4) and $\mathrm{H} 143$ (on a5), which were substituted for cysteine in a crosslinking assay (Figure 4), are also shown. (B) Superimposition of the helices a4-a5 from B. subtilis Noc $\triangle C T D-N B S$ complex upon the equivalent pair of helices in other apo-ParB/Noc or DNA-bound ParB structures (left panel), or upon the equivalent pair of helices in other nucleotide-bound ParB/Noc structure (right panel). The G. thermoleovorans NocN $\Delta 26 \Delta$ CTD-SO4 ${ }^{2-}$ structure is thought to represent the conformation of Noc in a nucleotide-bound state as the sulfate anion from crystallization solution occupies a position equivalent to that of the $\beta$-phosphate moiety of CTP 6 . 
A
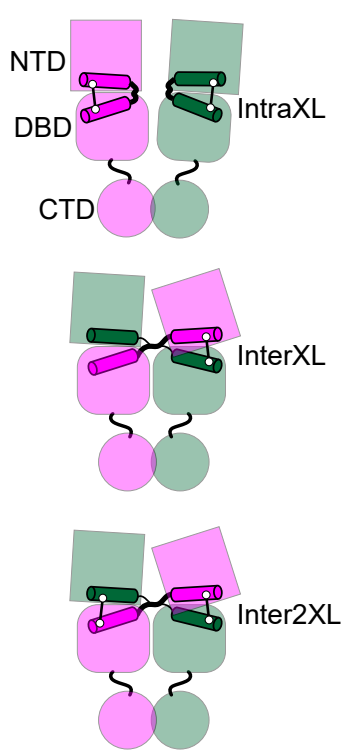

B
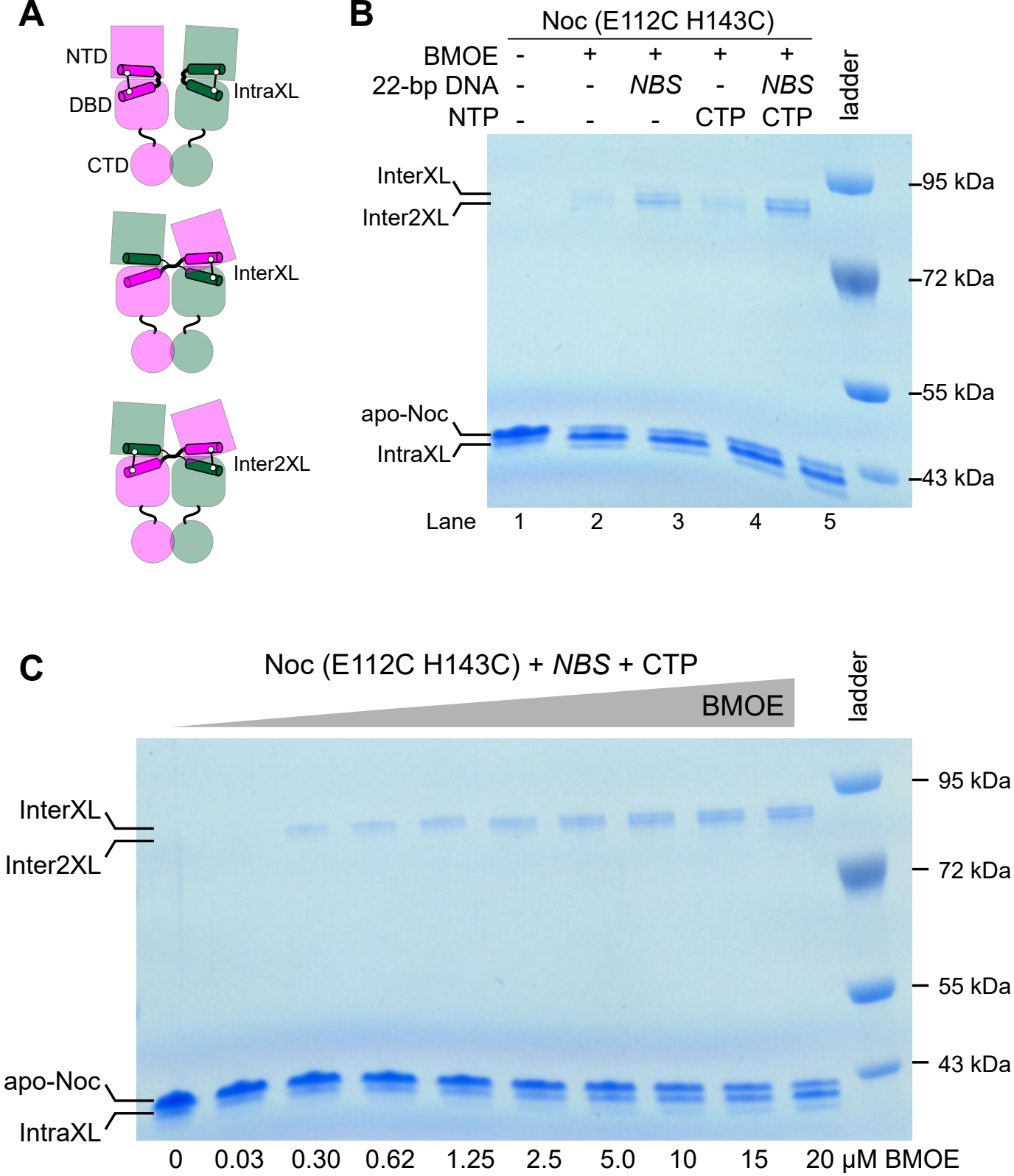

Figure 4. Site-specific crosslinking of $B$. subtilis Noc (E112C H143C) suggests that the swinging-out conformation of Noc-NBS exists in solution. (A) Schematic diagram of crosslinked species: IntraXL and InterXL/Inter2XL denote crosslinked species formed between $\alpha 4$ and $\alpha 5$ from either the same Noc subunit or from opposing subunits, respectively. Inter2XL represents a double crosslinking between $\alpha 4$ and $\alpha 5$ ', and between $\alpha 4$ ' and $\alpha 5$. (B) Crosslinking of Noc (E112C H143C) in the presence or absence of NBS and CTP and combinations thereof. Crosslinked species were resolved on an acrylamide gel and stained with Coomassie. Each crosslinking experiment was run in triplicate. (C) Crosslinking of $B$. subtilis Noc (E112C H143C) + CTP + NBS with an increasing concentration of BMOE. Crosslinked species were resolved on an acrylamide gel and stained with Coomassie. The top-most band appeared first as the concentration of BMOE is increasing from left to right, and thus it is thought to represent the singly crosslinked InterXL species. The doubly crosslinked Inter2XL presumably is more compacted and migrated faster than the Inter $X \mathrm{~L}$ on a denaturing acrylamide gel. Each crosslinking experiment was run in triplicate. 
A

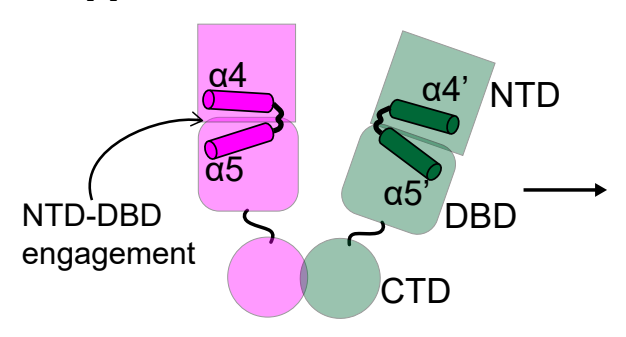

open clamp
$B_{i}$
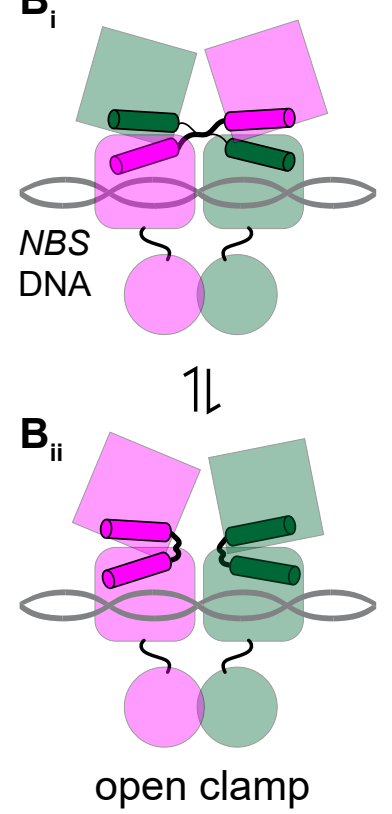

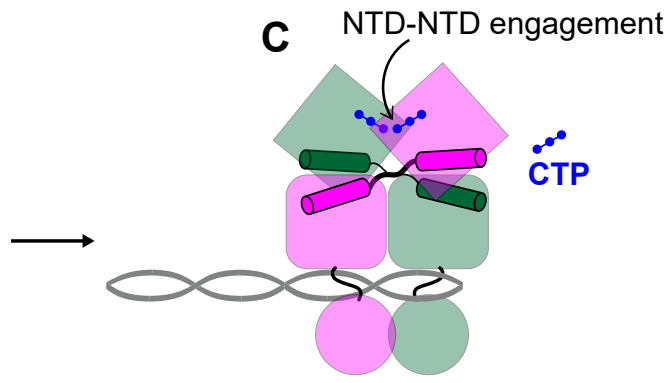

closed clamp

Figure 5. A possible model of different conformations of $B$. subtilis Noc. In apo-Noc (A), helices $\alpha 4$ and $\alpha 5$ from the same Noc subunit (magenta or dark green) likely pack together i.e. the foldingback conformation ${ }^{6}$. In NBS-bound Noc, helices $\alpha 4$ and $a 5$ from the same Noc subunit are not packed together, instead $\alpha 4$ swings outwards to pack against $\alpha 5^{\prime}$ from the adjacent Noc subunit i.e. the swinging-out conformation $\left(\mathrm{B}_{\mathrm{i}}\right)$. As the result, the NTD and the DBD from the same Noc subunit disengage from each other, and there is no protein-protein contact between the majority of two adjacent NTDs of Noc. The proximity of adjacent Noc subunits and the restriction in movement by a DNA-bound DBD may increase the likelihood of helix swapping, this might contribute to promoting the NTD-NTD engagement upon CTP-binding (C). It is possible that helices $\alpha 4$ and $\alpha 5$ of NBS-bound Noc also take up a folding-back conformation ( $\left.B_{i i}\right)$. In this case, NTDs of adjacent subunits of Noc likely adopt different orientations to avoid possible clashing between two adjacent protein subunits ${ }^{15}$, the difference in orientations of opposing NTDs has been observed in the co-crystal structures of ParB $\Delta$ CTD-parS from C. crescentus and H. pylori ${ }^{11,12}$. 


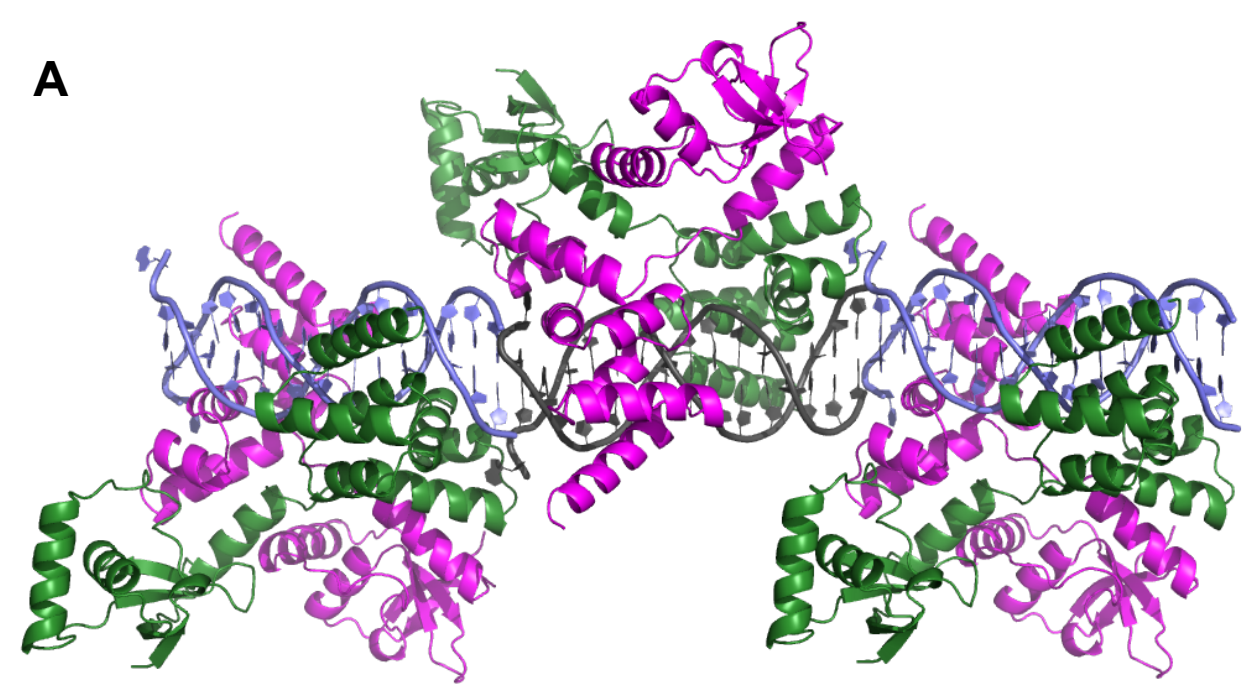

B

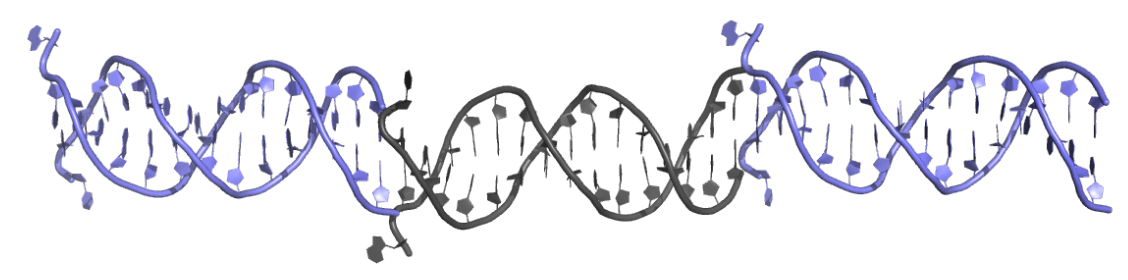

Supplementary Figure 1. (A) Co-crystal structure of $B$. subtilis Noc $\triangle C T D-N B S$ complex in the context of the crystal. Neighboring DNA duplexes (light blue and grey) stack and interact to form pseudo-continuous DNA filaments running through the crystal. (B) Only the NBS DNA portion from the Noc $\triangle$ CTD-NBS complex is shown for clarity. Note the flipped-out bases at the junctions between the duplexes. 


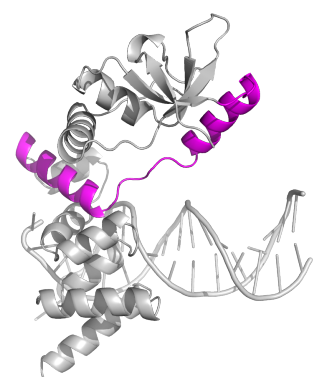

B. subtilis Noc $\triangle$ CTD-NBS (PDB: 7OL9)

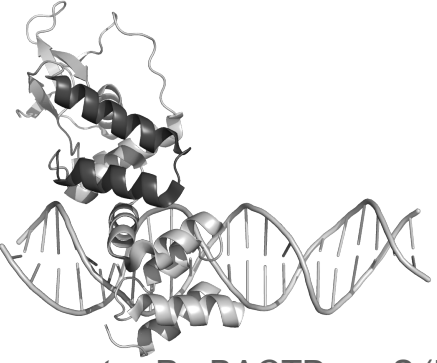

C. crescentus ParB $\Delta$ CTD-parS (PDB: 6T1F)

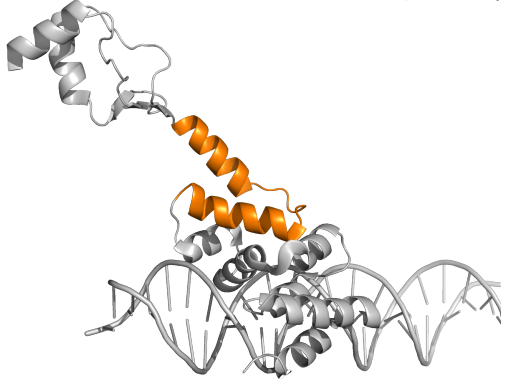

H. pylori ParB $\triangle$ CTD-parS (PDB: 4UMK)

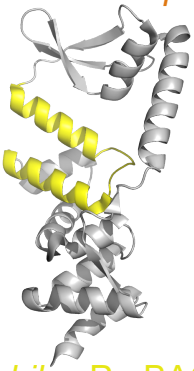

T. thermophilus ParB $\triangle$ CTD apo (PDB: 1VZO)

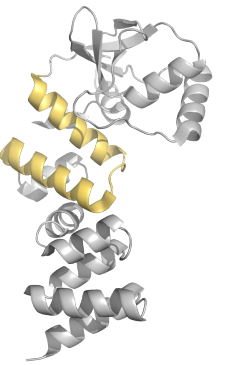

G. thermoleovorans Noc $\triangle \mathrm{CTD}$ apo (PDB: 7NFU)

the folding-back conformation of helices $\alpha 4-5$

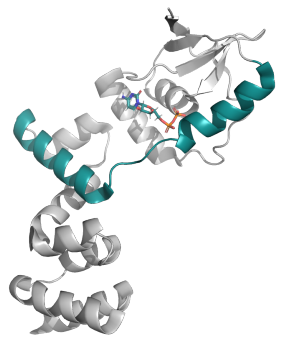

B. subtilis ParB $\triangle$ CTD-CDP (PDB: 6SDK)

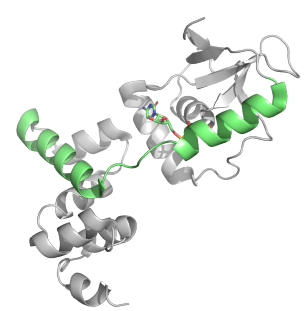

C. crescentus ParB $\triangle$ CTD-CTPyS (PDB: 7BM8)

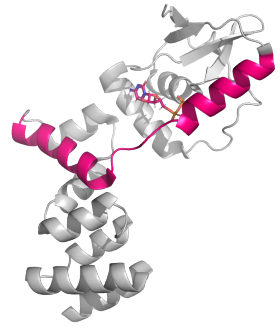

M. xanthus ParB $\triangle$ CTD-CDP (PDB: 7BNK)

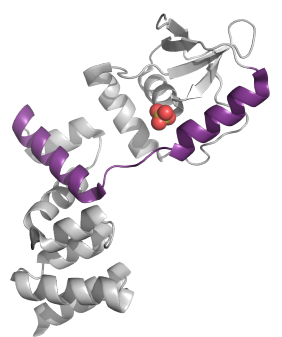

G. thermoleovorans Noc $\Delta$ N26 $\Delta$ CTD-SO42- (PDB: 7NG0) the swinging-out conformation of helices $\alpha 4-5$

Supplementary Figure 2. Structures of available chromosomal ParB and Noc, either in the apo-, DNA-bound, or nucleotide-bound states. Only the pair of helices $\alpha 4$ and $\alpha 5$ are highlighted in bright colors to show either the folding-back (left column) or the swinging-out conformation (right column). 

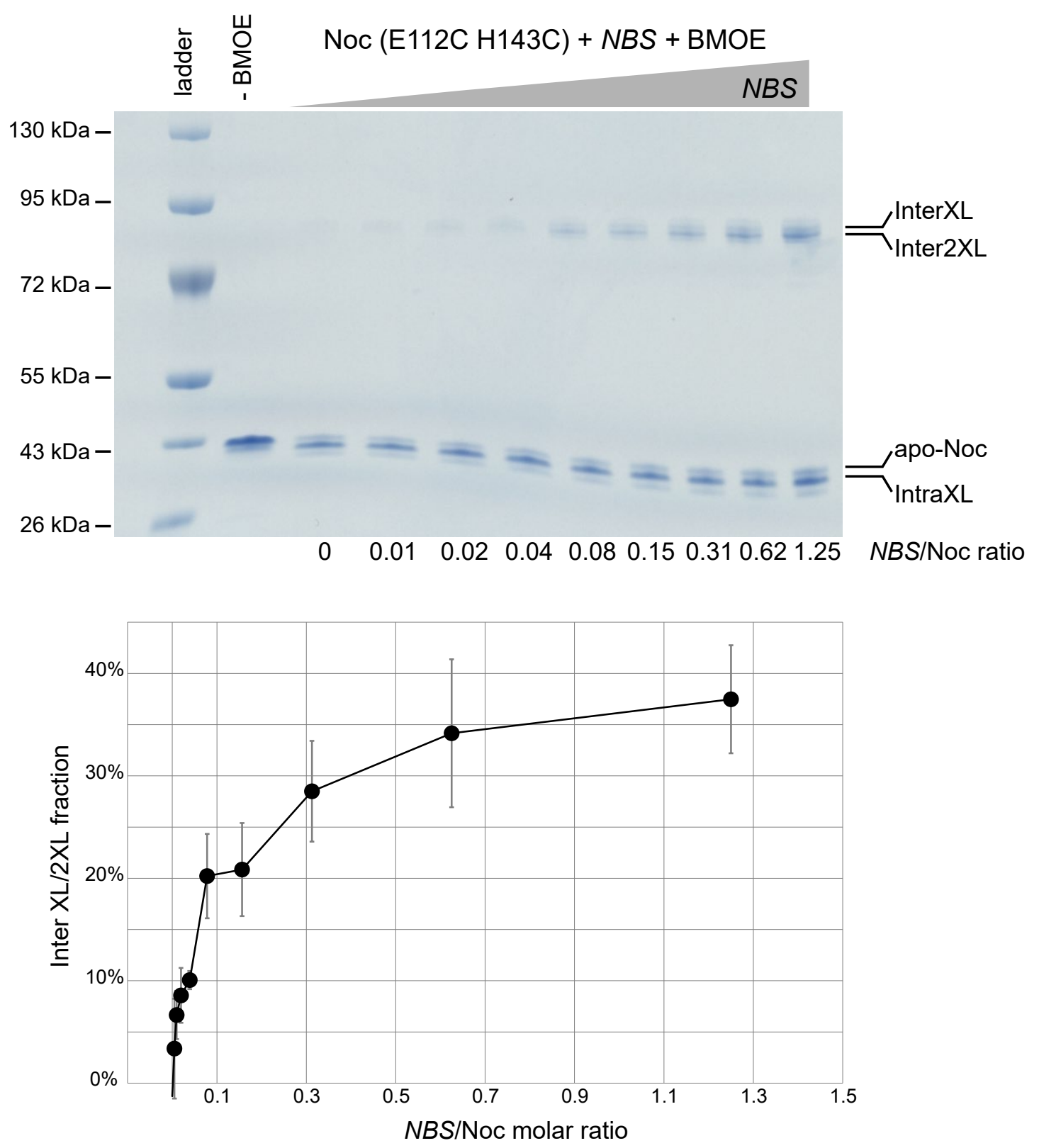

Supplementary Figure 3. Crosslinking of $B$. subtilis Noc (E112C H143C) in the presence of an increasing concentration of NBS and absence of CTP. Crosslinked species were resolved on an acrylamide gel and stained with Coomassie, and InterXL/2XL fractions were quantified. Each crosslinking experiment was run in triplicate. 